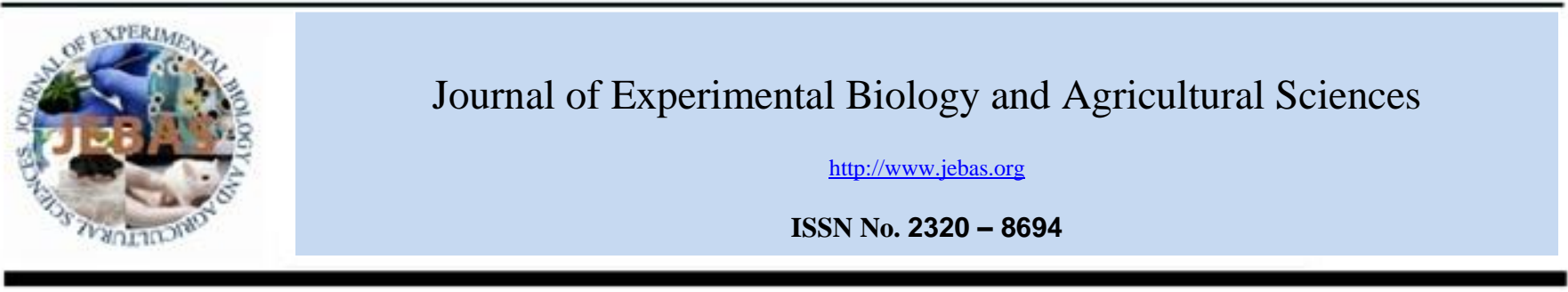

\title{
NUTRITIONAL QUALITY OF MAIZE IN RESPONSE TO DROUGHT STRESS DURING GRAIN-FILLING STAGES IN MEDITERRANEAN CLIMATE CONDITION
}

\author{
Celaleddin Barutçular ${ }^{1, *}$, Halef Dizlek ${ }^{2}$, Ayman EL-Sabagh ${ }^{3}$, Tulin Sahin $^{2}$, Mabrouk Elsabagh $^{4}$ and \\ Mohammad Shohidul Islam ${ }^{5}$
}

\author{
${ }^{1}$ Department of Field Crops, Faculty of Agriculture, University of Cukurova, 01330 Adana,Turkey \\ ${ }^{2}$ Department of Food Engineering, Faculty of Engineering, University of Osmaniye Korkut Ata, Turkey \\ ${ }^{3}$ Department of Agronomy, Faculty of Agriculture, Kafrelsheikh University, 33516 Kafr El-Sheikh, Egypt \\ ${ }^{4}$ Department of Nutrition and Clinical Nutrition, Faculty of Veterinary Medicine, Kafr El-sheikh University, 33516 Kafr El-Sheikh, Egypt \\ ${ }^{5}$ Department of Agronomy, University of Hajee Mohammad Danesh Science and Technology, Bangladesh
}

Received - September 24, 2016; Revision - October 19, 2016; Accepted - October 16, 2016

Available Online - November 13, 2016

DOI: http://dx.doi.org/10.18006/2016.4(Issue6).644.652

\begin{abstract}
KEYWORDS
Deficit irrigation

Grain filling stage

Starch

Oil and protein content

Zea mays

ABSTRACT

Maize is considered one of the most essential dietary components in human food and animal feeding. The objectives of the present study were to quantify the effects of drought stress on qualitative traits of maize at grain-filling stages. Hybrids maize seeds were grown by applying full and water stress conditions during the grain filling stage. Various nutritional properties (crude oil, starch, grain protein content) were determined in 2014 and 2015 at the second crop growing season in Adana, Turkey. Based on the results of this study, genotype and environment were found to influence all quality traits significantly. Further, result of study suggest that water stress caused a significant reduction in major quality traits. Grain weight and grain quality yield as well crude oil, protein and ash yield were significantly decreased due to water deficit condition in the both growing seasons. Significant differences were observed among hybrids in respect of all measurements due to irrigation regimes. The genotypes, Sancia and Calgary were tolerant by producing higher grain weight. Accordingly, grain qualities of 71May69, Aaccel and Calgary maize hybrids were less affected under drought stress.
\end{abstract}

* Corresponding author

E-mail: cebar@cu.edu.tr (Celaleddin Barutçular)

Peer review under responsibility of Journal of Experimental Biology and Agricultural Sciences.

Production and Hosting by Horizon Publisher India [HPI] (http://www.horizonpublisherindia.in/).

All rights reserved.
All the article published by Journal of Experimental Biology and Agricultural Sciences is licensed under a Creative Commons Attribution-NonCommercial 4.0 International License Based on a work at www.jebas.org.

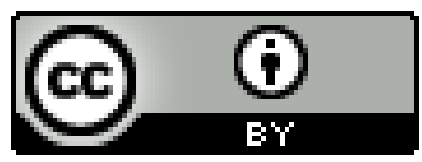




\section{Introduction}

Maize (Zea mays) is an important food and feed crop for human and livestock, and the demand of maize production is increasing day by day due to its multipurpose uses which include medicine and textile industies as well as biofuel production (White \& Johnson 2003; Ali et al., 2010). As a temperate zone country, Turkey produces about 5.9 million tons of maize per year and it is cultivated in approximately 0.66 million hectares (FAOSTAT, 2014). About 35\% of Turkish maize production is used for human consumption, $65 \%$ for animal feed (Kusaksiz, 2010).

Water shortage was extended in the crop production area. Hence, Food, feed and industrial demant of quality properties have gradually increasing in maize grain. Maize is a sensitive crop to water stress and it's growth is negatively affected by unavailability of water at its growing stages (Byrne et al., 1995). Drought might be severely reduced the various qualitative trait such as grain starch, granule size and increased relative protein content (Balla et al., 2011). Drought stress increased is shortened the grain filling period and reduced grain yield, grain weight and specific weight (Gooding et al., 2003). According to Zhao et al. (2009) maize protein components are very sensitive to drought stress during grain filling stage. The degradation in the dough quality could be due to the decline in the glutenin to gliadin ratio and in the percentage of very large glutenin polymers in response to a biotic stress (Balla \& Veisz, 2007).

Maize production program has been primarily aimed for increasing yield, quality and stability under different environments (Ignjatovic-Micic et al., 2014). Therefore, grain yield is the most commonly studied parameters, but grain quality parameters had less attention for hybrids. Rehman et al. (2011) reported grain protein, oil and starch content of maize are generally stable in different environments. Storage components of mature kernel of maize as a quality traits, starch, protein and oil content are determinators of the final grain weight (Boyer \& Hannah, 2001). Based on the above context, the objectives of the currentstudy was to elucidate the effect of drought stress on maize grain weight and, as well as nutritional properties (grain starch, protein, crude oil, ash and yield) for seven maize hybrids under deficit irrigation in Mediterranean climate condition.

\section{Materials and Methods}

\subsection{Experimental design and cultural practices}

Field trials were conducted in growing season of 2014 and 2015 as second crop maize at research field of Cukurova University, Adana, Turkey. A summary of climatic data are given in figure 1.The methodologies have been followed as described previously by EL Sabagh et al. (2015). The experimental design used in this study was strip-split plot in four replications. The materials were consist of (1) 7 hybrids variety of maize (Sancia, Indaco, 71May69, Aaccel, Calgary, 70May82 and 72May80) and, (2) two moisture levels (Full irrigation and water stress ) and amount of irrigation are given in Figure 1 and treatments were applied at grain growth stages. Hybrids were sown during first and the second year on 28 June, 2014 and 12 June, 2015, respectively. The regular agronomic practices of growing maize were similar to farmers' practice and were followed as necessary. During experiments, nitrogenous fertilizer was utilized within two times of planting, $100 \mathrm{~kg} \mathrm{~N}$ and P2O5 ha-1 (20-20-0) and V6-growth stage 200 kg N ha-1 (Urea).

\subsection{Measurements}

Proximate composition of grain including protein, starch, oil and ash were analyzed based on the method prescribed by $\operatorname{AACC}(2000)$.

Table 1 Effects of analysis of variance of maize hybrids under irrigation regimes in both seasons

\begin{tabular}{|c|c|c|c|c|c|c|c|c|c|c|}
\hline $\begin{array}{l}\text { Source of } \\
\text { variation }\end{array}$ & $\mathrm{GW}(\mathrm{mg})$ & TW(kg/hl) & $\mathrm{SC}(\%)$ & $\mathrm{PC}(\%)$ & $\mathrm{OC}(\%)$ & $\mathrm{AC}(\%)$ & $\begin{array}{c}\text { SY } \\
\text { (kg/ ha) }\end{array}$ & $\begin{array}{c}\text { PY } \\
\text { (kg/ha) }\end{array}$ & $\begin{array}{c}\text { OY } \\
\text { (kg/ha) }\end{array}$ & $\begin{array}{c}\text { AY } \\
(\mathrm{kg} / \mathrm{ha})\end{array}$ \\
\hline \multicolumn{11}{|l|}{2014} \\
\hline Hybrids & $* *$ & $*$ & ns & ns & $* * *$ & $* *$ & $* *$ & $* * *$ & ns & $* *$ \\
\hline Interaction & $\mathrm{ns}$ & $\mathrm{ns}$ & $\mathrm{ns}$ & $*$ & $* *$ & $*$ & ns & $* * *$ & $* *$ & $* *$ \\
\hline $\mathrm{CV} \%$ & 10.7 & 2.9 & 5.0 & 6.0 & 6.7 & 6.6 & 8.1 & 8.8 & 7.7 & 9.8 \\
\hline \multicolumn{11}{|l|}{2015} \\
\hline Irrigation & $*$ & $\mathrm{~ns}$ & $\mathrm{~ns}$ & $\mathrm{~ns}$ & ns & $* *$ & $* *$ & $*$ & $*$ & $\mathrm{~ns}$ \\
\hline Hybrids & $* *$ & $* *$ & $* * *$ & ns & $*$ & $* *$ & ns & ns & $* *$ & ns \\
\hline Interaction & $* *$ & ns & $* * *$ & $\mathrm{~ns}$ & $\mathrm{~ns}$ & ns & $*$ & $\mathrm{~ns}$ & $* *$ & $\mathrm{~ns}$ \\
\hline $\mathrm{CV} \%$ & 5.2 & 1.8 & 3.8 & 4.6 & 11.6 & 6.5 & 8.5 & 7.6 & 14.0 & 9.0 \\
\hline
\end{tabular}

ns: Indicates nonsignificant; $*, * *$ and $* * *, \quad$ significant $P<0.05, P<0.01$ and $P<0.001$ probability respectively; GW: grain weight,TW:testweight (kg/hl),SC:starch content (\%), PC:protein content (\%), OC:oil content (\%),AC:ash content (\%), SY:starch yield (kg/ha), PY:protein yield (kg/ha), OY:oil yield (kg/ha) and AY:ash yield (kg/ha). 


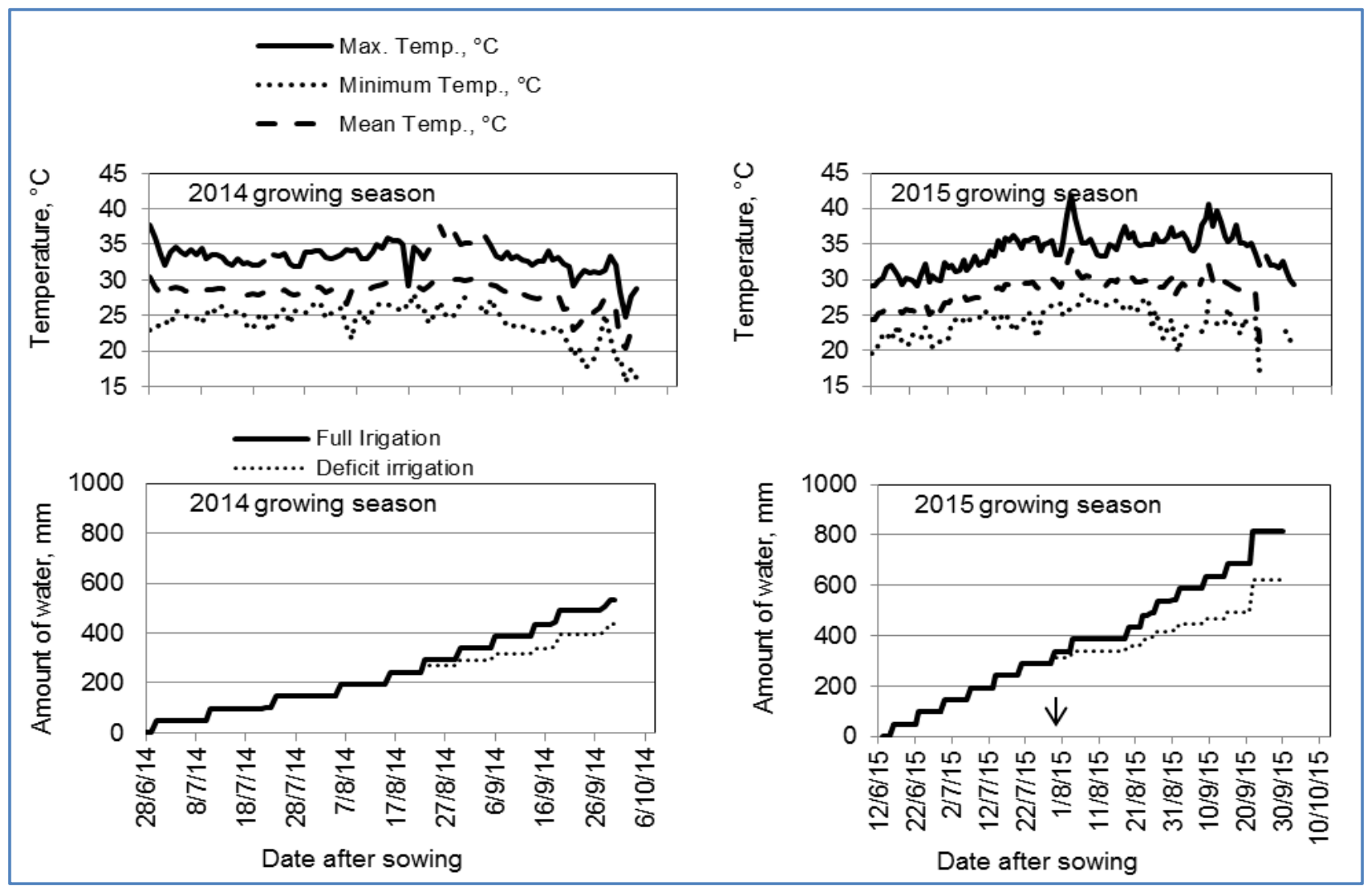

Figure 1 Temperature and amount of water during 2014 and 2015 growing season (Arrow indicates pollination) (Source: Meteorological Service of Turkish State, 2016).

Table 2 Irrigation regimes effects on grain quality parameters of maize hybrids in 2014 and 2015.

\begin{tabular}{|c|c|c|c|c|c|c|c|c|c|c|}
\hline Traitments & GW(mg) & $\begin{array}{c}\text { TW } \\
(\mathrm{kg} / \mathrm{hl})\end{array}$ & $\begin{array}{c}\mathbf{S} \\
\mathbf{C}(\%)\end{array}$ & $\mathrm{PC}(\%)$ & $\mathrm{OC}(\%)$ & $\mathrm{AC}(\%)$ & $\begin{array}{c}\text { SY(kg/ } \\
\text { ha) }\end{array}$ & $\begin{array}{c}\text { PY } \\
\text { (kg ha) }\end{array}$ & $\begin{array}{c}\text { OY } \\
\text { (kg/ha) }\end{array}$ & $\begin{array}{c}\mathrm{AY} \\
(\mathbf{k g} / \mathbf{h a})\end{array}$ \\
\hline \multicolumn{11}{|l|}{2014 growing season } \\
\hline Irrigated (Control) & 258 & 77.9 & 62.8 & 7.66 & 2.77 & 1.08 & 8060 & 983 & 355 & 138 \\
\hline Deficit irrigated & 232 & 77.9 & 62.5 & 8.01 & 2.79 & 1.02 & 6596 & 849 & 294 & 108 \\
\hline Drought reduction & -0.10 & 0.00 & 0.00 & 0.05 & 0.01 & -0.06 & -0.18 & -0.14 & -0.17 & -0.22 \\
\hline Probability & ns & ns & ns & ns & ns & $*$ & $* * *$ & $*$ & $* *$ & $* * *$ \\
\hline \multicolumn{11}{|l|}{2015 growing season } \\
\hline Irrigated(Control) & 292 & 72.2 & 64.0 & 8.05 & 2.63 & 1.07 & 9159 & 1150 & 376 & 152 \\
\hline Deficit irrigated & 275 & 72.0 & 64.1 & 8.09 & 2.60 & 1.05 & 8069 & 1020 & 328 & 133 \\
\hline Drought reduction & -0.06 & 0.00 & 0.00 & 0.01 & -0.01 & -0.01 & -0.12 & -0.11 & -0.13 & -0.13 \\
\hline Probability & $*$ & $\mathrm{~ns}$ & ns & $\mathrm{ns}$ & $\mathrm{ns}$ & $\mathrm{ns}$ & $* *$ & $* *$ & $*$ & $*$ \\
\hline
\end{tabular}

ns: Indicates nonsignificant; $*, * *$ and $* * *, \quad$ significant $P<0.05, P<0.01$ and $P<0.001$ probability respectively; GW: grain weight,TW:testweight (kg/hl),SC:starch content (\%),PC:protein content (\%),OC:oil content (\%),AC:ash content (\%),SY:starch yield(kg/ha),PY:protein yield(kg/ha),OY:oil yield(kg/ha) and AY:ash yield(kg/ha). 
Table 3 Maize hybrids grain quality traits and quality yield parameters in 2014 and 2015 growing season.

\begin{tabular}{|c|c|c|c|c|c|c|c|c|c|c|}
\hline Hybrids & $\begin{array}{l}\text { GW } \\
(\mathbf{m g})\end{array}$ & $\begin{array}{c}\text { TW } \\
(\mathrm{kg} / \mathrm{hl})\end{array}$ & $\begin{array}{l}\text { SC } \\
(\%)\end{array}$ & $\begin{array}{l}\text { PC } \\
(\%)\end{array}$ & $\begin{array}{l}\text { OC } \\
(\%)\end{array}$ & $\begin{array}{l}\text { AC } \\
(\%)\end{array}$ & $\begin{array}{c}\text { SY } \\
(\mathbf{k g} / \mathbf{h a})\end{array}$ & $\begin{array}{c}\text { PY } \\
\text { (kg/ha) }\end{array}$ & $\begin{array}{c}\text { OY } \\
\text { (kg/ha) }\end{array}$ & $\begin{array}{c}\text { AY } \\
(\mathrm{kg} / \mathrm{ha})\end{array}$ \\
\hline \multicolumn{11}{|c|}{2014 growing season } \\
\hline H1 & 236 & 76.3 & 64.5 & 7.99 & 2.56 & 0.96 & 8219 & 1020 & 326 & 123 \\
\hline $\mathrm{H2}$ & 271 & 78.6 & 63.0 & 7.85 & 2.85 & 1.07 & 7379 & 925 & 332 & 126 \\
\hline H3 & 232 & 78.5 & 64.2 & 8.11 & 2.80 & 1.09 & 7912 & 1000 & 345 & 134 \\
\hline H4 & 263 & 76.7 & 60.6 & 7.59 & 2.83 & 1.06 & 6973 & 869 & 324 & 122 \\
\hline H5 & 223 & 76.6 & 62.3 & 7.63 & 2.60 & 1.09 & 7401 & 901 & 307 & 129 \\
\hline H6 & 257 & 78.6 & 61.0 & 8.05 & 3.01 & 1.06 & 6658 & 879 & 327 & 117 \\
\hline H7 & 234 & 80.1 & 62.9 & 7.62 & 2.80 & 1.01 & 6757 & 820 & 309 & 109 \\
\hline Mean & 245 & 77.9 & 62.6 & 7.84 & 2.78 & 1.05 & 7328 & 916 & 324 & 123 \\
\hline LSD $_{0.05}$ & 26.7 & 2.3 & ns & ns & 0.187 & 0.072 & 601.6 & 81.2 & ns & 12.3 \\
\hline \multicolumn{11}{|c|}{2015 growing season } \\
\hline H1 & 247 & 70.1 & 63.8 & 8.47 & 2.59 & 1.08 & 8715 & 1155 & 353 & 147 \\
\hline $\mathrm{H2}$ & 294 & 73.6 & 63.6 & 7.93 & 2.67 & 1.04 & 7632 & 957 & 321 & 126 \\
\hline H3 & 290 & 72.7 & 65.2 & 8.54 & 2.52 & 1.03 & 9386 & 1232 & 365 & 152 \\
\hline H4 & 298 & 71.9 & 64.9 & 7.78 & 2.64 & 1.05 & 8965 & 1073 & 366 & 145 \\
\hline H5 & 253 & 69.5 & 62.4 & 7.86 & 2.62 & 1.14 & 7988 & 1005 & 336 & 147 \\
\hline H6 & 327 & 73.1 & 65.2 & 7.94 & 2.68 & 1.02 & 9484 & 1155 & 391 & 147 \\
\hline H7 & 275 & 73.7 & 63.2 & 7.98 & 2.58 & 1.05 & 8132 & 1019 & 332 & 135 \\
\hline Mean & 283 & 72.1 & 64.0 & 8.07 & 2.61 & 1.06 & 8614 & 1085 & 352 & 143 \\
\hline $\mathbf{L S D}_{0.05}$ & 15.0 & 1.32 & ns & 0.379 & ns & 0.072 & 746.1 & 83.4 & ns & 13.0 \\
\hline
\end{tabular}

ns: Indicates nonsignificant; GW: grain weight,TW:testweight (kg/hl),SC:starch content (\%), PC:protein content (\%), OC:oil content (\%),AC:ash content (\%), SY:starch yield (kg/ha), PY:protein yield (kg/ha), OY:oil yield (kg/ha) and AY:ash yield (kg/ha); H1:Sancia ,H2:Indaco,H3:71May69, H4:Aaccel,H5:Calgari, H6:70May82 and H7:72May80

For grain weight determination randomly 10 ears were selected and shelling, it was followed by weighed of grain to calculate the percentage of shelling through (grain weight/grain numbers) at $12.5 \%$ moisture level of grain.

\subsection{Statistical analysis}

The obtained results subjected to analyses of variance according to Gomez \& Gomez (1984). Significant means were separated by the Least Significant Difference (LSD) at the 0.05 significance level $(\mathrm{P} \leq 0.05)$. The estimation of correlation for traits was calculated by MSTAT-C computer software package.

\section{Results and Discussion}

\subsection{Effects of irrigation regimes on quality traits of maize}

Effect of irrigation regimes was the most prominent source which affects the grain quality during various growth stages (Table $1 \& 2$ ). It was reported that grain quality of maize hybrids were significantly influenced by irrigation treatments and, water stress lead to a significant reduction in yield quality traits over control (Table $1 \& 2$ ). Amount of maize oil, starch, protein and ash yield were significantly reduced by the deficit irrigation, starch showed higher sensitivity to drought
$(\mathrm{P}<0.001$ and $\mathrm{P}<0.01$ of first and second year, respectively) than other traits (Table 2).

It was observed that grain weight was significantly affected by water stress and the highest grain weight (258 and $292 \mathrm{mg}$ ) was observed under control while the lowest grain weight (232 and $275 \mathrm{mg}$ ) under water stress condition for the 1st and 2nd year respectively (Table 2). Low grain weight due to drought stress, as found in present experiments, may indicate that the plants were unable to fully meet the demand of the growing grain. In present research, the differences between the water regimes were statistically significant for ash content in first season (Table 2). Protein content is also significantly influenced by water stress conditions and, it was slightly increased (non-significant) under limited irrigations (Table 2).

High starch, protein and crude oil yielding genotypes under water shortage condition could be evaluated for the drought tolerance genotypes. The obtained results, oil yield per unit area considerably decreased under water stress (Table 2). Grain qualities are governed by a number of factors particularly the duration and rate of grain filling (Brdar et al., 2008) and availability of assimilates that are negatively influenced under water deficit conditions (Ali \& Ashraf, 2011; Barutçular et al., 2016a). 
Table 4 Grain quality traits of maize hybrids as influenced by water regimes in 2014 and 2015.

\begin{tabular}{|c|c|c|c|c|c|c|c|c|c|c|}
\hline Hybrids & $\begin{array}{l}\text { GW } \\
(\mathbf{m g})\end{array}$ & $\begin{array}{l}\text { TW } \\
(\mathrm{kg} / \mathrm{hl})\end{array}$ & $\begin{array}{l}\text { SC } \\
(\%)\end{array}$ & $\begin{array}{l}\text { PC } \\
(\%)\end{array}$ & $\begin{array}{l}\text { OC } \\
(\%)\end{array}$ & $\begin{array}{l}\mathrm{AC} \\
(\%)\end{array}$ & $\begin{array}{l}\text { SY } \\
\text { ( kg/ ha) }\end{array}$ & $\begin{array}{l}\text { PY } \\
\text { (kg/ha) }\end{array}$ & $\begin{array}{l}\text { OY } \\
\text { (kg/ha) }\end{array}$ & $\begin{array}{l}\text { AY } \\
\text { (kg/ha) }\end{array}$ \\
\hline \multicolumn{11}{|c|}{ Irrigated (Control) in 2014} \\
\hline H1 & 255 & 76.8 & 66.7 & 7.65 & 2.66 & 0.97 & 8481 & 972 & 338 & 123 \\
\hline $\mathrm{H} 2$ & 282 & 78.9 & 63.0 & 8.18 & 2.66 & 1.09 & 8177 & 1061 & 344 & 142 \\
\hline H3 & 237 & 77.6 & 62.3 & 7.92 & 2.77 & 1.06 & 8525 & 1084 & 378 & 146 \\
\hline H4 & 282 & 76.6 & 60.2 & 7.18 & 2.71 & 1.11 & 7833 & 935 & 352 & 144 \\
\hline H5 & 229 & 76.8 & 63.9 & 7.15 & 2.54 & 1.10 & 8306 & 929 & 330 & 142 \\
\hline H6 & 266 & 77.7 & 61.6 & 7.96 & 2.92 & 1.16 & 7198 & 930 & 340 & 136 \\
\hline H7 & 257 & 80.8 & 61.8 & 7.57 & 3.15 & 1.06 & 7903 & 969 & 402 & 135 \\
\hline \multicolumn{11}{|c|}{ Deficit irrigated (Water stress) in 2014} \\
\hline H1 & 217 & 75.7 & 62.2 & 8.33 & 2.45 & 0.96 & 7957 & 1067 & 314 & 124 \\
\hline H2 & 260 & 78.3 & 63.0 & 7.52 & 3.05 & 1.05 & 6582 & 789 & 320 & 110 \\
\hline H3 & 227 & 79.4 & 66.2 & 8.31 & 2.83 & 1.11 & 7299 & 915 & 311 & 122 \\
\hline H4 & 244 & 76.7 & 61.0 & 8.00 & 2.95 & 1.00 & 6113 & 803 & 296 & 101 \\
\hline H5 & 217 & 76.5 & 60.8 & 8.11 & 2.66 & 1.08 & 6496 & 872 & 284 & 117 \\
\hline H6 & 249 & 79.4 & 60.4 & 8.14 & 3.11 & 0.96 & 6117 & 829 & 315 & 98 \\
\hline H7 & 211 & 79.5 & 64.1 & 7.67 & 2.45 & 0.96 & 5610 & 672 & 215 & 84 \\
\hline $\mathbf{L S D}_{0.05}$ & ns & $\mathrm{ns}$ & ns & 0.676 & 0.264 & 0.101 & $\mathrm{~ns}$ & 114.9 & 36.0 & 17.3 \\
\hline \multicolumn{11}{|c|}{ Irrigated (Control) in 2015} \\
\hline H1 & 240 & 70.0 & 64.1 & 8.70 & 2.60 & 1.05 & 8978 & 1216 & 363 & 147 \\
\hline H2 & 302 & 73.2 & 64.3 & 8.50 & 2.74 & 1.07 & 8254 & 1094 & 351 & 138 \\
\hline H3 & 303 & 72.3 & 63.2 & 8.39 & 2.56 & 1.09 & 10056 & 1337 & 409 & 174 \\
\hline H4 & 305 & 71.9 & 64.9 & 7.66 & 2.70 & 1.03 & 9543 & 1126 & 397 & 151 \\
\hline H5 & 260 & 70.7 & 61.1 & 7.55 & 2.56 & 1.14 & 8338 & 1027 & 349 & 155 \\
\hline H6 & 357 & 73.4 & 65.6 & 7.76 & 2.66 & 1.01 & 9699 & 1149 & 393 & 149 \\
\hline H7 & 279 & 73.8 & 65.0 & 7.80 & 2.59 & 1.08 & 9248 & 1104 & 368 & 154 \\
\hline \multicolumn{11}{|c|}{ Deficit irrigated (Water stress) in 2015} \\
\hline H1 & 253 & 70.1 & 63.5 & 8.25 & 2.59 & 1.11 & 8452 & 1094 & 343 & 148 \\
\hline H2 & 286 & 74.0 & 62.9 & 7.36 & 2.60 & 1.00 & 7009 & 821 & 292 & 114 \\
\hline H3 & 277 & 73.1 & 67.2 & 8.69 & 2.48 & 0.98 & 8716 & 1128 & 322 & 130 \\
\hline H4 & 291 & 71.9 & 64.9 & 7.90 & 2.58 & 1.08 & 8386 & 1020 & 334 & 139 \\
\hline H5 & 247 & 68.3 & 63.8 & 8.17 & 2.68 & 1.15 & 7638 & 984 & 324 & 138 \\
\hline H6 & 298 & 72.9 & 64.9 & 8.12 & 2.70 & 1.03 & 9269 & 1161 & 389 & 145 \\
\hline H7 & 271 & 73.7 & 61.4 & 8.17 & 2.58 & 1.03 & 7015 & 934 & 296 & 116 \\
\hline $\mathbf{L S D}_{0.05}$ & 21.2 & $\mathrm{~ns}$ & $\mathrm{~ns}$ & 0.54 & $\mathrm{~ns}$ & $\mathrm{~ns}$ & $\mathrm{~ns}$ & 117.9 & $\mathrm{~ns}$ & 18.4 \\
\hline
\end{tabular}

ns: Indicates nonsignificant; GW: grain weight,TW:testweight (kg/hl),SC:starch content (\%), PC:protein content (\%), OC:oil content (\%),AC:ash content (\%), SY:starch yield (kg/ha), PY:protein yield (kg/ha), OY:oil yield (kg/ha) and AY:ash yield (kg/ha).H1:Sancia ,H2:Indaco,H3:71May69, H4:Aaccel,H5:Calgari, H6:70May82 and H7:72May80

Water stress imposed during the grain filling period of wheat, especially at the early filling stage, usually results in a reduction in grain weight (Zhao et al., 2009). Further, Pierre et al. (2008) and EL Sabagh et al. (2015) reported that water deficit stress has a negative effect on grain weight. Protein content is more strongly influenced by environment (Mikhaylenko et al., 2000).Variations in flour quality in a hard-grained were related to changes in protein composition from drought stress during grain filling (Gooding et al., 2003). It was also reported that water limitation significantly decreases seed and oil yields of maize (Ghassemi-Golezani \& Dalil, 2011).The reduction in protein and oil yields under water stress could be due to sharp decline in grain yield under stressful condition (Ghassemi-Golezani \& Lotfi, 2013) and the results are also the results are also in agreement with the findings of Rashwan et al. (2016) and Barutçular et al. (2016b).

\subsection{Comparative evaluation of various hybrids of maize}

Significant differences among various genotypes with respect to grain quality traits were observed which indicates existence of genetic variation and possibility of selection for favorable genotypes in both environments. In this research, a greater reduction in test weight was observed in genotype of Calgari and Sancia (Table 1, 3). The hybrids genotype Indaco, 70May82 and Aaccel showed more positive effect of grain weight. 
Table 5 Pearson correlation coefficient between grain quality traits in the irrigation regime (2014 and 2015 growing season).

\begin{tabular}{|c|c|c|c|c|c|c|c|c|c|c|}
\hline Traits & $\begin{array}{l}\text { GW } \\
(\mathbf{m g})\end{array}$ & $\begin{array}{c}\text { TW } \\
(\mathrm{kg} / \mathrm{hl})\end{array}$ & $\begin{array}{l}\mathrm{SC} \\
(\%)\end{array}$ & $\begin{array}{l}\text { PC } \\
(\%)\end{array}$ & $\begin{array}{l}\text { OC } \\
(\%)\end{array}$ & $\begin{array}{l}\mathrm{AC} \\
(\%)\end{array}$ & $\begin{array}{c}\text { SY } \\
(\mathrm{kg} / \mathrm{ha})\end{array}$ & $\begin{array}{c}\text { PY } \\
\text { (kg/ha) }\end{array}$ & $\begin{array}{c}\text { OY } \\
\text { (kg/ha) }\end{array}$ & $\begin{array}{c}\mathrm{AY} \\
(\mathrm{kg} / \mathrm{ha})\end{array}$ \\
\hline \multicolumn{11}{|c|}{ Irrigated (Control) in 2014} \\
\hline GW (mg) & 1.000 & & & & & & & & & \\
\hline TW (kg/hl) & 0.161 & 1.000 & & & & & & & & \\
\hline $\mathrm{SC}(\%)$ & -0.389 & -0.236 & 1.000 & & & & & & & \\
\hline $\mathrm{PC}(\%)$ & 0.266 & 0.370 & 0.072 & 1.000 & & & & & & \\
\hline $\mathrm{OC}(\%)$ & 0.150 & $0.769^{*}$ & -0.437 & 0.211 & 1.000 & & & & & \\
\hline $\mathrm{AC}(\%)$ & 0.249 & 0.003 & -0.750 & 0.017 & 0.126 & 1.000 & & & & \\
\hline SY (kg/ha) & -0.485 & -0.178 & 0.604 & -0.076 & -0.516 & -0.749 & 1.000 & & & \\
\hline PY (kg ha) & -0.027 & 0.261 & 0.082 & 0.681 & -0.068 & -0.288 & 0.545 & 1.000 & & \\
\hline $\mathrm{OY}(\mathrm{kg} / \mathrm{ha})$ & -0.066 & 0.733 & -0.399 & 0.097 & $0.782 *$ & -0.157 & 0.032 & 0.330 & 1.000 & \\
\hline $\mathrm{AY}(\mathrm{kg} / \mathrm{ha})$ & -0.013 & -0.033 & -0.690 & -0.051 & -0.158 & 0.612 & -0.014 & 0.308 & 0.137 & 1.000 \\
\hline \multicolumn{11}{|c|}{ Deficit irrigated (Water stress) in 2014} \\
\hline GW (mg) & 1.000 & & & & & & & & & \\
\hline TW (kg/hl) & 0.194 & 1.000 & & & & & & & & \\
\hline $\mathrm{SC}(\%)$ & -0.262 & 0.497 & 1.000 & & & & & & & \\
\hline $\mathrm{PC}(\%)$ & -0.351 & -0.311 & -0.052 & 1.000 & & & & & & \\
\hline $\mathrm{OC}(\%)$ & $0.929 * *$ & 0.320 & -0.250 & -0.161 & 1.000 & & & & & \\
\hline $\mathrm{AC}(\%)$ & 0.055 & 0.055 & 0.412 & 0.102 & 0.213 & 1.000 & & & & \\
\hline SY ( kg/ ha) & -0.179 & -0.460 & 0.252 & 0.624 & -0.247 & 0.242 & 1.000 & & & \\
\hline PY (kg ha) & -0.202 & -0.590 & -0.041 & $0.789 *$ & -0.218 & 0.112 & $0.939 * *$ & 1.000 & & \\
\hline OY(kg/ha) & 0.608 & -0.257 & -0.187 & 0.399 & 0.595 & 0.283 & 0.607 & 0.634 & 1.000 & \\
\hline $\mathrm{AY}(\mathrm{kg} / \mathrm{ha})$ & -0.085 & -0.508 & 0.142 & 0.600 & -0.071 & 0.563 & $0.904 * *$ & $0.870^{*}$ & 0.684 & 1.000 \\
\hline \multicolumn{11}{|c|}{ Irrigated (Control) 2015} \\
\hline GW (mg) & 1.000 & & & & & & & & & \\
\hline TW (kg/hl) & 0.704 & 1.000 & & & & & & & & \\
\hline $\mathrm{SC}(\%)$ & 0.544 & 0.592 & 1.000 & & & & & & & \\
\hline $\mathrm{PC}(\%)$ & -0.289 & -0.209 & 0.075 & 1.000 & & & & & & \\
\hline $\mathrm{OC}(\%)$ & 0.468 & 0.371 & 0.564 & 0.077 & 1.000 & & & & & \\
\hline $\mathrm{AC}(\%)$ & -0.572 & -0.283 & $-0.890 * *$ & -0.090 & -0.589 & 1.000 & & & & \\
\hline SY ( kg/ ha) & 0.491 & 0.256 & 0.436 & -0.073 & -0.207 & -0.475 & 1.000 & & & \\
\hline PY (kg ha) & 0.098 & -0.076 & 0.141 & 0.588 & -0.293 & -0.219 & 0.718 & 1.000 & & \\
\hline OY(kg/ha) & 0.582 & 0.239 & 0.379 & -0.092 & -0.047 & -0.478 & $0.959 * *$ & 0.690 & 1.000 & \\
\hline $\mathrm{AY}(\mathrm{kg} / \mathrm{ha})$ & 0.012 & -0.057 & -0.352 & -0.092 & -0.722 & 0.355 & 0.646 & 0.625 & 0.619 & 1.000 \\
\hline \multicolumn{11}{|c|}{ Deficit irrigated (Water stress) 2015} \\
\hline GW (mg) & 1.000 & & & & & & & & & \\
\hline TW (kg/hl) & $0.758^{*}$ & 1.000 & & & & & & & & \\
\hline $\mathrm{SC}(\%)$ & 0.270 & -0.030 & 1.000 & & & & & & & \\
\hline $\mathrm{PC}(\%)$ & -0.331 & -0.242 & 0.516 & 1.000 & & & & & & \\
\hline $\mathrm{OC}(\%)$ & -0.024 & -0.385 & -0.341 & -0.354 & 1.000 & & & & & \\
\hline $\mathrm{AC}(\%)$ & -0.668 & $-.931 * *$ & -0.239 & 0.028 & 0.507 & 1.000 & & & & \\
\hline SY ( kg/ha) & 0.303 & -0.118 & $0.759^{*}$ & 0.516 & 0.050 & -0.018 & 1.000 & & & \\
\hline PY (kg/ha) & 0.073 & -0.205 & 0.676 & 0.753 & -0.015 & 0.048 & $0.941 * *$ & 1.000 & & \\
\hline OY(kg/ha) & 0.268 & -0.226 & 0.439 & 0.292 & 0.484 & 0.192 & $0.888 * *$ & $0.821^{*}$ & 1.000 & \\
\hline AY (kg/ha) & -0.159 & -0.633 & 0.428 & 0.392 & 0.346 & 0.574 & 0.798* & $0.782 *$ & $0.844 *$ & 1.000 \\
\hline
\end{tabular}

*, **, significant $\quad P<0.05$ and $P<0.01$ probability respectively; GW: grain weight,TW:testweight $(\mathrm{kg} / \mathrm{hl}), \mathrm{SC}$ :starch content $(\%)$, PC:protein content (\%), OC:oil content (\%),AC:ash content (\%), SY:starch yield (kg/ha), PY:protein yield (kg/ha), OY:oil yield (kg/ha) and AY:ash yield ( $\mathrm{kg} / \mathrm{ha})$. 
Achieved results revealed that oil content was significantly influenced by water regime and that maximum value was found for genotype 70May82 (3.01\%) and minimum for Sancia $(2.56 \%)$ in the first season. The obtained results also revealed that the highest value of protein content $(8.54 \%)$ was found in 71 May69 and the lowest $(7.78 \%)$ in Aaccel. In this experiment, genotype Calgari produced the highest value of grain ash content in both seasons. The hybrids Indaco, 71May69 and Sancia showed more positive effects on starch and protein yield/ha. Results of study revealed that ash yield was significantly influenced by water stress conditions and that the maximum value of ash (134 kg/ha) was found in 71May69 and the minimum $(109 \mathrm{~kg} / \mathrm{ha})$ in 72 May80. Drought stress reduced grain weight but increased protein content in wheat as reported by Rharrabti et al. (2003). Availability of assimilates are negatively influenced under water deficit conditions (Ali \& Ashraf, 2011). Water deficient at flowering stage greatly decreased starch content due to the decrease of photosynthesis and thus makes an increase of grain protein ratio (Mousavi et al., 2013). Environment is the major source of variation for grain quality as reported by Mikhaylenko et al. (2000); Gulluoglu et al. (2016) and Kurt et al.(2016).

3.3 Effects of maize hybrids and irrigation regimes on quality traits

Genotypes and irrigation regimes were found to influence all quality traits significantly. The treatments interactions showed significant differences on the quality parameters (Table 4). Under drought stress, the hybrids Sancia and Calgary were more stable in grain weight (less reduction) and 71may69, Aaccel and Calgary were less sensitive to grain quality (less grain quality losses), maize quality properties are usually influenced by genotypes, environmental factors and their interactions and final growth stage of maize is dramatically influenced by the water stress and, this adverse effects are mainly reduced grain weight, and this resulted in low starch, crude oil and protein content (Cirilo et al., 2011). Grain filling process is sensitive to environmental conditions, this strongly influencing the final grain development quantitatively and qualitatively as well (Yang \& Zhang, 2006). Mansouri et al. (2010) found that, grain weight were decreased under water stress condition. Farhad et al. (2013) observed that grain protein and oil contents were significantly influenced by irrigation regimes among maize hybrids.

\subsection{Correlation analysis}

Correlation coefficients among the major studied variables were found positive association in both seasons (Table 5). It was found that the grain weight was negatively affected by the starch content in the first season, and protein content and ash content in the second season. The highest correlation was observed in starch, protein yield in both seasons, while, negative correlation between oil content and starch content as well as protein content was found in the first season. It was also observed a negative correlation among grain weight, test weight and ash in the second season. Oury \& Godin (2007) reported that, protein contents were negatively correlated with grain weight under normal and stress conditions. The negative correlation between grain yield and grain gluten content had been established under genotype-by-environment interactions in different studies of wheat (Tayyar, 2010). The positive correlation between grain protein percentage and grain filling rate and which results from irrigation-off at flowering stage, has a significant effect on grain protein percentage increasing. Furthermore, it was observed that, grain protein percentage reduces in relation to starch under water deficient (Mousavi et al., 2013).

\section{Conclusion}

In summary, it can be stated that the imposition of water stress significantly influenced the nutritional quality traits of maize. However, high starch, protein and crude oil yielding genotypes could be evaluated for the drought tolerance in the drought environment. In respect to the hybrids, Sancia and Calgary were drought tolerant genotypes for their less sensitivity (more stable) to grain weight under drought stress. The 71May69, Aaccel and Calgary were less sensitive in grain quality traits under drought stress.

\section{Conflict of interest}

Authors would hereby like to declare that there is no conflict of interests that could possibly arise.

\section{References}

AACC, American Association of Cereal Chemists (2000) International approved methods of analysis (10th ed.). AACC International, St. Paul, MN, U.S.A.

Ali Q, Ashraf M (2011) Exogenously applied glycinebetaine enhances seed and seed oil quality of maize (Zea mays L.) under water deficit conditions. Environmental and Experimental Botany 71: 249-259. doi: 10.1016/j.envexpbot.2010.12.009.

Ali Q, Ashraf M, Anwar F (2010) Seed composition and seed oil antioxidant activity of maize under water stress. Journal of the American Oil Chemists' Society 87: 1179-1187. doi: 10.1007/s11746-010-1599-5.

Balla K, Rakszegi M, Li Z, Békés F, Bencze S, Veisz O (2011) Quality of winter wheat in relation to heat and drought shock after anthesis. Czech Journal of Food Sciences 29: 117-128.

Balla K, Veisz O (2007) Changes in the quality of cereals in response to heat and drought stress. Acta Agronomica Óvariensis 49: 451-455.

Barutçular C, Yıldırım M, Koç M, Akıncı C, Toptaş I, Albayrak O, Tanrikulu A, EL Sabagh A (2016a) Evaluation of SPAD chlorophyll in spring wheat genotypes under different 
environments. Fresenius Environmental Bulletin 25:12581266.

Barutçular C, Yıldırım M, Koç M, Akıncı C, Tanrıkulu A, ELSabagh A, Saneoka H, Ueda A, Islam MS, Toptas I, Albayrak O, Tanrikulu A (2016b) Quality traits performance of bread wheat genotypes under drought and heat stress conditions. Fresenius Environmental Bulletin 25 : 1-7.

Boyer CD, Hannah LC (2001) Kernel mutants of corn. In: Hallauer AR (Eds.) Specialty corns. CRC, Boca Raton, FL. pp. $1-31$.

Brdar MD, Kraljevíc-Balalíc MM, Kobiljski BD (2008) The parameters of grain filling and yield components in common wheat (Triticum aestivum L.) and durum wheat (Triticum turgidum L.var. durum). Central European Journal of Biology 3:75-82. doi:10.2478/s11535-007-0050-x.

Byrne PF, Bolanos J, Edmeades GO, Eaton DL (1995) Gains from selection under drought versus multilocation testing in related tropical maize populations. Crop Science 35: 63-69. doi:10.2135/cropsci1995.0011183X003500010011x.

Cirilo AG, Actis M, Andrade FH, Valentinuz OR (2011) Crop management affects dry-milling quality of Flint maize kernels. Field Crops Research 122, 140-150.

EL Sabagh A, Barutçular C, Saneoka H (2015) Assessment of drought tolerance maize hybrids at grain growth stage in Mediterranean area. International Journal of Biological, Biomolecular, Agricultural, Food and Biotechnological Engineering 9: 989-992.

FAOSTAT, Food and Agriculture Organization of The United Nations Statistics Division (2016) Available on http://faostat3.fao.org/download/Q/QC/E Access date: 15.08.2016.

Farhad W, Cheema MA, Saleem MF, Radovich T, Abbas F, Hammad HM, Wahid MA (2013)Yield and quality response of maize hybrids to composted poultry manure at three irrigation levels. International Journal of Agriculture \& Biology 15:181190.

Ghassemi-Golezani K, Dalil B (2011) Seed ageingand field performance of maize under waterstress. African Journal of Biotechnology 10: 18377-18380. doi: 10.5897/AJB11.2154.

Ghassemi-Golezani K, Lotfi R (2013) Influence of water stress and pod position on oil and protein accumulation in soybean grains. International Journal of Agronomy and Plant Production 4: 2341-2345.

Gooding MJ, Ellis RH, Shewry PR (2003) Effects of restrictedwater availability and increased temperature on the grain filling, drying and quality of winter wheat. Journal of Cereal Science 37: 295-309. doi:10.1006/jcrs.2002.0501.
Gomez KA, Gomez AA (1984) Statistical procedures for agricultural research. John Wiley and Sons. New York. pp. 680 .

Gulluoglu L, Bakal H, Onat B, EL Sabagh A, Arioglu H (2016) Characterization of peanut (Arachishypogaea L.) seed oil and fatty acids composition under different growing season under Mediterranean environment. Journal of Experimental Biology and Agricultural Sciences 4 (Suppl. V) : 564571.DOI: http://dx.doi.org/10.18006/2016.4 (5S).564.571

Ignjatovic-Micic D, Kostadinovic M, Bozinovic $\mathrm{S}$, Andjelkovic V, Vancetovic J (2014) High grain quality accessions within a maize drought tolerant core collection. Scientia Agricola 71:345-355.

Kurt C, Arioglu H, Erdem T, Akkaya MR, El Sabagh A, salam MS (2016) A comparative study of fatty acid extraction methods of sesame (Sesamum indicum L.) Varieties grown under Mediterranean environment. Journal of Experimental Biology and Agricultural Sciences 4 (Suppl. V) : 589-593. (2016).DOI: http://dx.doi.org/10.18006/2016.4(5S).588.593.

Kusaksiz T (2010) Adaptability of some new maize (Zea mays L.) cultivars for silage production as main crop in Mediterranean environment. Turkish Journal of Field Crops, 15: 193-197.

Mansouri-Far CSA, Sanavy MM, Saberali SF (2010) Maize yield response to deficit irrigation during low sensitive growth stages and nitrogen rate under semi-arid climatic conditions. Agricultural Water Management 97:12-22.

Mousavi H, Lack S, Alavi Faze M (2013) Analysis of Correlation and Stepwise Regression between Grain Protein Yield and Related Traits of Maize in Conditions of Drought Stress and Zinc Sulfate Spraying. International Journal of Agriculture and Crop Sciences 5:2783-2788.

Mikhaylenko GG, Czuchajowska Z, Baik BK, Kidwell KK (2000) Environmental influences on flour composition, dough rheology and baking quality of spring wheat. Cereal Chemistry 77: 507-511. doi: 10.1094/CCHEM.2000.77.4.507.

Oury F, Godin C (2007) Yield and grain protein concentration in bread wheat: how to use the negative relationship between the two characters to identify favourable genotypes? Euphytica 157: 45-57. doi:10.1007/s10681-007-9395-5.

Pierre CS, Peterson CJ, Ross AS, Ohm J, Verhoeven MC, Larson M, White BH (2008) Wheat grain quality changes with genotype, nitrogen fertilization, and water stress. Agronomy Journal 100: 414-420. doi:10.2134/agrojnl2007.0166.

Rashwan E, Mousa A, EL-Sabagh A, Barutçular C( 2016) Yield and Quality Traits of Some Flax Cultivars as Influenced by Different Irrigation Intervals. Journal of Agricultural 
Science $\quad 8 \quad:$ 226-240.DOI:

http://dx.doi.org/10.5539/jas.v8n10p226.

Rehman A, Saleem MF, Safdar ME, Hussain S, Akhtar N (2011) Grain quality, nutrient use efficiency, and bioeconomics of maize under different sowing methods and NPK levels. Chilean Journal of Agricultural Research 71: 586-593. doi:10.4067/S0718-58392011000400014.

Rharrabti Y, Royo C, Villegas D, Aparicio N, Garcia LF (2003) Durum wheat quality in Mediterranean environments I: Quality expression under different zones, latitudes, and water regimes across Spain. Field Crops Research 80: 123-131. doi: Field Crops Research.

Tayyar S (2010) Variation in grain yield and quality of Romanian bread wheat varieties compared to local varieties in northwestern Turkey. Romanian Biotechnological Letters 15: 5189-5196.

White PJ, Johnson LA (2003) Corn: chemistry and technology.pp.892. Ann.Ass.Cereal Chem. Inc. St. Paul, Mn, USA.

Yang JC, Zhang JH (2006) Grain filling of cereals under soil drying. New Phytologist 169: 223-236. doi: 10.1111/j.14698137.2005.01597.x.

Zhao CX, He MR, Wang ZL, Wang YF, Lin Q (2009) Effects of different water availability at post-anthesis stage on grain nutrition and quality in strong-gluten winter wheat. Comptes Rendus Biologies 332: 759-764. doi: 10.1016/j.crvi.2009.03.003. 